\title{
Swirling Flows Characteristics in a Cylinder Under Effect of Buoyancy
}

\author{
Mustafa FEKHAR*, Rachid SACI*, Renée GATIGNOL** \\ *Lab. “L.D.M.V”, University of Boumerdes, Boumerdes 35000, Algeria, E-mail: r.saci@univ-boumerdes.dz \\ (Corresponding Author), E-mail:fekharm@univ-boumerdes.dz. \\ **Institut Jean Le Rond D’Alembert, UPMC \& CNRS, Paris 05, France, E-mail: renee.gatignol@upmc.fr \\ crossref http://dx.doi.org/10.5755/j02.mech.27975
}

\section{Introduction}

Swirling flows have been investigated extensively aiming at providing further insight into the conditions that cause abrupt flow stagnation and associated breakdown. This latter complex feature may be evidenced, for instance, in the benchmark model flow driven by one end disk of an upright cylinder of given aspect ratio $[1,2]$. Several prior studies provided means of controlling breakdown onset as it can be harmful / beneficial, depending upon the application considered. Hereafter, are presented selected investigations based on kinematic, geometric and thermal means of vortex flows control.

Of note, Mullin et al. [3] demonstrated the effects of including a tapered center body and showed it to alter significantly breakdown onset and location. In particular, they argued that the inhibition / suppression of the vortex structure depended on the axial pressure gradient sign, induced by the conical axial rod, whose circular base is sealed to either the rotating or the stationary disk of the enclosure. Manungua et al. [4], through numerical simulations and dye visualizations, attributed the effective vortex control to the role of vorticity strength, modified upstream of the vortex by the differential rotation of a partial rotating disk flash mounted into the top lid. More recently, breakdown topology in an enclosure shrouded by a polygonal cross section sidewall was explored numerically and experimentally by Naumov et al. [5]. Despite the drastic change in the lateral geometry, the findings revealed that the core flow which displays breakdown remains axisymmetric and the 3D non axisymmetric behavior was remarked in the bulk flow adjacent to the sidewall. The topology and parameter dependence of breakdown was addressed by Jones et al. [6]. Their analysis led to the identification of the adequate parameters which allowed consistent comparison between bubble breakdown observed in a torsionally driven cylinder flow and the spiral type vortex occurring in flows through openended pipes. More recently, Shtern [7] highlighted the role of the swirl decay as a physical mechanism to control breakdown development.

Thermal effects on confined vortex flows were addressed numerically by, for instance, Lugt et al. [8]. Their findings evidenced the sensitivity of breakdown to weak buoyancy caused by an unstable thermal stratification resulting from a slightly heated bottom disk. Under stable thermal stratification, significant effects on the swirling flow topology were reported by Omi et al. [9] who extended the investigation to account for co-/counter-rotating end disks. Compressibility effects were demonstrated by Herrada et al. $[10,11]$, in the case of a non-Boussinesq swirling flow driven by the bottom disk rotation of a cylinder with and without a central rod. A combination of buoyancy and rod differential rotation provided efficient means of vortex flows control: a forced axial temperature gradient was shown to enhance or eliminate the vortex features; depending on the sign of the temperature gradient. Flow stagnation and associated breakdown which occur in a swirling jet under effect of thermal buoyancy was addressed by Dina et al. [12] who performed PIV measurements and concluded that a temperature gradient between the jet core and its surrounding fluid causes the suppression/enhancement of the vortex pattern; depending on the sign of the gradient.

Effects of a density variation on a confined isothermal swirling flow which exhibits on-axis vortex breakdown, driven by the top disk rotation of a cylinder, were recently addressed experimentally by Ismadi et al. [13] using axial injection of dye, denser or lighter than the ambient liquid. Flow visualizations indicated that heavy dye enhanced the formation of breakdown while very light dye injection (density variations of order $0.01 \%$ ) caused the occurrence of offaxis ring type vortices and modified the threshold of breakdown onset.

Concerns over the symmetry/asymmetry of onaxis breakdown in a cylinder were noted by several investigators: dye visualizations often provide images of vortices with asymmetric folds while 3D numerical counterparts, under well controlled conditions, predict axisymmetric patterns $[6,14,15]$. This discrepancy is often attributed to the inevitable imperfections of the set up and operating conditions such as a $0.01 \%$ misalignment of the cavity axis [14] or a slight tilt $\left(\approx 2^{\circ}\right)$ of one end disk [15].

Motivated by the above considerations, the current work explored numerically the sensitivity of a helical flow and associated vortex characteristics to a differential heating in a disk-cylinder system. Buoyancy was first implemented by a warm/cool axial fluid injection then by means of a differentially heated/cooled tiny rod.

\section{Formulation and method of solution}

\subsection{Governing parameters}

We consider a vertical cylinder of height $H$ and radius $R$, filled with an incompressible viscous Newtonian fluid, whose top disk rotates with a uniform angular velocity $\Omega$ as sketched in Fig.1. The set up includes, at the bottom disk center, either a circular orifice of radius ratio $\delta_{o}=R_{o} / R$ to allow uniform axial fluid injection or a small rod of equal radius ratio $\delta_{r}=R_{r} / R=\delta_{0}$ with an aspect ratio $\delta_{h}=R_{r} / h$. The flows under consideration are assumed to be laminar and axisymmetric which reduces the numerical domain to a meridian plane as illustrated in Fig. 1 which includes a typical on-axis bubble type breakdown and selected 
vortex characteristics.

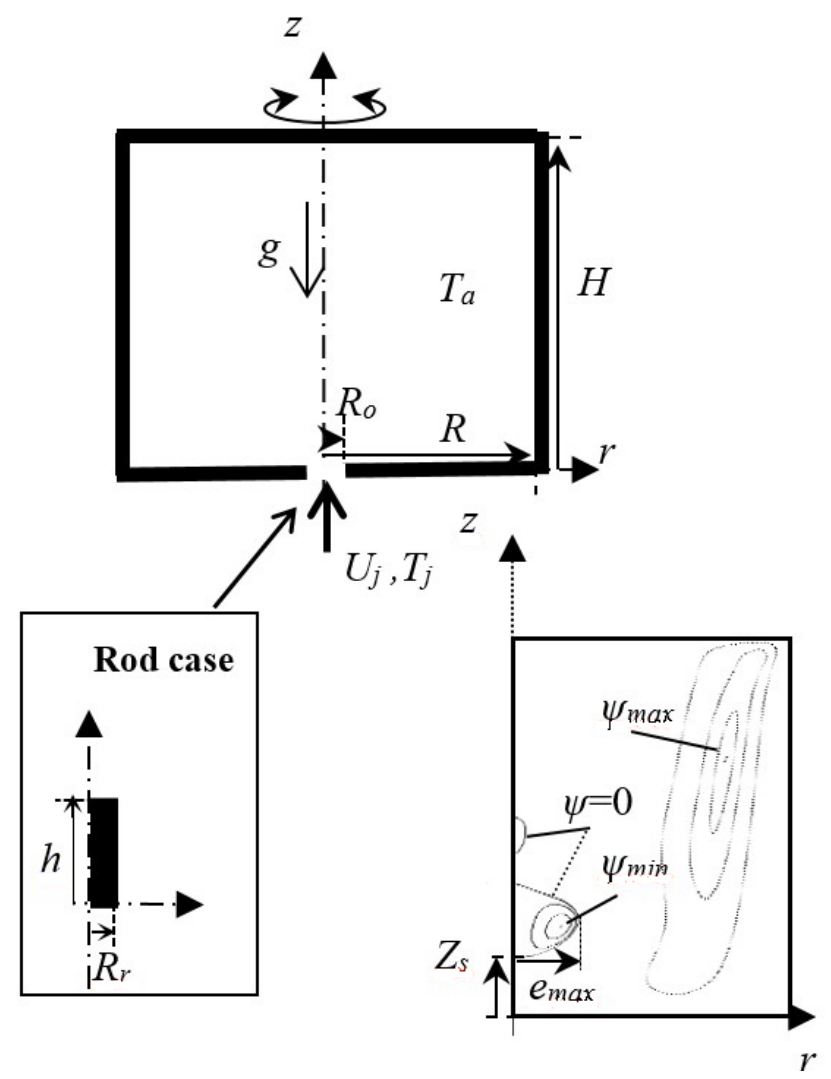

Fig. 1 Schematic of the geometry and numerical domain which displays a meridian streamline pattern with an on-axis two bubble type breakdown and selected vortex characteristics: $Z_{s}$ (upstream stagnation point), $e_{\max }$ (bubble radial extent), stagnation line $\psi=0$ maximum volumetric flow rates inside $\left(\psi_{\min }<0\right)$ and outside $\left(\psi_{m a c}>0\right)$ the vortex

The flow is conveniently described in cylindrical coordinates $(r, \varphi, z)$. Using $1 / \Omega, R$ and $\rho_{a}$ as time, length and density scales respectively, all variables and flow fields are written in dimensionless form. The non-dimensional velocity field (scale: $\Omega R$ ) is expressed in terms of the stream function $\psi(r, z)$ (scale: $\Omega R^{3}$ ) and circulation $\Gamma=v r$ (scale: $\Omega R^{2}$ ) in order to provide an interpretation of the local meridian flow direction $(u, v, w)=\left(\frac{1}{r} \frac{\partial \psi}{\partial z}, \frac{\Gamma}{r}, \frac{1}{r} \frac{\partial \psi}{\partial r}\right)$.

The main parameters, required to govern the dynamics of the flows under consideration are: the rotational Reynolds, the cavity aspect ratio and the Richardson number which appropriately accounts for thermal buoyancy strength, defined respectively as:

$$
R e=\frac{\Omega R^{2}}{v}, \Lambda_{h}=\frac{H}{R}, R i=\frac{\beta g \Delta T}{\Omega^{2} R},
$$

where: $\Delta T=T j-T a$ is the temperature gradient between the jet and the ambient fluid; $v$ and $\beta$ denote the kinematic viscosity and thermal expansion coefficients respectively.

\subsection{Initial and boundary conditions}

The well documented isothermal flow driven by the top disk rotation, in the absence of injection/rod, which displays a steady bubble type breakdown for a given couple of parameters $\left(R e, \Lambda_{h}\right)$, is defined as a basic state.

At $t=0$, injection is applied $\left(U_{j} \neq 0, T_{j} \neq T_{a}\right)$ and maintained over the time range $0 \leq t \leq t_{f} ; t_{f}$ being dependent on the configuration considered. In general, $t_{f} \leq 300$ (which corresponds to a maximum injected fluid volume ratio $<0.05 \%$ ) was found sufficient to capture the main vortex transient behavior over the range of parameters considered.

Boundary conditions which apply for all times $\left(0 \leq t \leq t_{f}\right)$ no slip and adiabatic conditions at all rigid walls and symmetry is assumed at the cavity axis. At the inlet orifice $\left(z=0,0 \leq r<\delta_{0}\right):(u, v, w ; T)=\left(0,0, U_{j} ; T_{j}\right)$.

\subsection{Method of solution}

The set of time dependent axisymmetric NavierStokes equations together with the mass conservation and energy equation [14], subjected to the above boundary conditions, were integrated under the Boussinesq approximation using a finite volume approach, implemented in the CFD package ANSYS Fluent 15. The laminar solver is adopted and the volume of fluid method is used when injection is considered. This code was recently employed successfully to accurately model confined as well as jet like swirling flows, which display breakdown, analogous to the configuration under consideration [16-18].

We used a segregated solver in which a second order upwind scheme was employed to discretize the convective terms, a first order implicit scheme was adopted for time marching while central differencing is utilized to approximate the diffusion terms. Pressure-velocity coupling was achieved by using the SIMPLEC algorithm and double precision was considered in all calculations.

The Convergence criteria were based on the asymptotic level reached by the scaled residuals of each equation. Overall, these were found to lie between $10^{-10}$ for the continuity and momentum equations and $10^{-15}$ for the swirl and energy equations.

The computational domain is the meridian plane $(r, z) \in] 0,1[\times] 0, \Lambda_{h}[$. An orthogonal grid, especially clustered in the viscous core region of the helical flow was utilized in order to adequately capture the requisite details of the vortex characteristics. The appropriate number of cells and associated time steps were determined on the basis of a grid sensitivity analysis. For the selected configuration with $\Lambda_{h}=1.98$ which is frequently referred to in this work, the grid sensitivity was evaluated by considering two sets of non-uniform grids, clustered along the cavity axis where breakdown is expected to occur. In particular, we used 45000 and 80000 cells along with a uniform grid of $200 \times 400$ elements in the $(r, z)$ plane for comparison.

Time marching was carried out using different time steps over the range $10^{-4} \leq \Delta t \leq 0.005$. It was found that, a non-uniform grid of 80000 cells associated to a time increment $\Delta t=0.001$ were sufficient to predict the main vortex characteristics with a maximum error $<1 \%$ compared to the solution predicted with the smallest time step $\Delta t=10^{-4}$. In addition, to speed up the convergence process and for computational efficiency, simulations were generally initialized with a solution from a lower $R e$. 


\section{Validation}

The numerical accuracy was first assessed by simulating the helical flow in the absence of injection, investigated experimentally by Escudier [1] and Iwatsu et al. [19] and supported quantitatively by numerous numerical predictions [2, 19].Validation was then extended to include the configuration under heavy dye injection [13].

\subsection{Prior configurations without injection}

It is well established that for the selected parameters $\left(R e, R i, \Lambda_{h}\right)=(1850,0,2)$, the bulk helical flow driven by the uniform rotation of one end disk of a vertical cylinder displays a primary large meridian circulation and two distinct on-axis bubble type vortices characterized by an upstream stagnation point located on the cavity axis at an axial distance $Z_{s}$ away from the non-rotating disk. The current calculations were found to reproduce qualitatively the flow topologies visualized in experiments $[1,19]$ and very accurately the numerical predictions $[2,19]$. In table 1 are reported three selected vortex flows characteristics (Fig.1); namely, the upstream (first) stagnation point location $\left(Z_{s}\right)$, the radial extent of the vortex pattern $\left(e_{\max }\right)$ scaled with the cylinder radius, as well as the vortex strengths $\psi_{\min }$ and $\psi_{\max }$ (meridian volume flow rates respectively within and outside the vortex structure). It is remarked that the maximum error estimate is within $0.5 \%$ on the stagnation point's location (sensitive criterion for onset /suppression of breakdown) and approximately $1 \%$ for the above volume flow rates which is considered as negligibly small.

Table 1

Values of the vortex characteristics: $Z_{s}, e_{\max }, \psi_{\min }$ and $\psi_{\max }$ obtained in this work and in [1, 19];

$\left(R e, R i, \Lambda_{h}\right)=(1850,0,2)$

\begin{tabular}{|c|c|c|c|c|}
\hline $\begin{array}{c}\text { Vortex } \\
\text { Characteristics }\end{array}$ & $\begin{array}{c}\text { Exp. } \\
\text { Escudier (1984) }\end{array}$ & $\begin{array}{c}\text { Num. } \\
\text { Iwatsu et al. (2007) }\end{array}$ & $\begin{array}{c}\text { Present } \\
\text { Work }\end{array}$ & $\begin{array}{c}\text { Error } \\
\text { Estimate }\end{array}$ \\
\hline$Z_{s}$ & 0.42 & 0.4231 & 0.4210 & $<0.5 \%$ \\
\hline$e_{\max }$ & 0.236 & 0.225 & 0.2264 & $<1.56$ \\
\hline$\psi_{\min }\left(10^{-4}\right)$ & - & -0.5916 & -0.5981 & $1 \%$ \\
\hline$\psi_{\max }\left(10^{-2}\right)$ & & 0.7832 & 0.7867 & $<0.5 \%$ \\
\hline
\end{tabular}

\subsection{Prior experiments under heavy dye injection}

Further comparison was carried out by considering the simulation of the above confined swirling flow subjected to heavy dye injection, applied at the bottom disk center, explored experimentally by Ismadi et al. [13]. To the best of our knowledge, there has been no prior numerical study of this model helical flow.

To allow direct comparison, we adopt the experimental conditions: $\Lambda_{h}=1.98, U_{j}=1.7 \times 10^{-3} \mathrm{~m} / \mathrm{s}$ while varying the density ratio $\Delta \rho / \rho_{a}=\left(\rho_{j}-\rho_{a}\right) / \rho_{a}\left(\rho_{a}\right.$ and $\rho_{j}$ are densities of the jet and ambient fluid respectively) over the range $0 \%<\Delta \rho / \rho_{a} \leq 0.02 \%$. The validation focused on the behavior of axisymmetric and non-oscillatory flow structures which limits the comparison to $R e \leq 2600$ [20].

Fig.2 illustrates the effect of injecting a fluid slightly heavier than the ambient one, i.e. $\rho_{a}>\rho_{j}$. Good agreement is observed between the present numerical predictions and experiments [12] as both reveal breakdown enhancement (decrease of $R e$ ) with increasing $\rho_{j}$ almost the same rate as depicted by the trend of the bounding curve which indicates first breakdown onset.

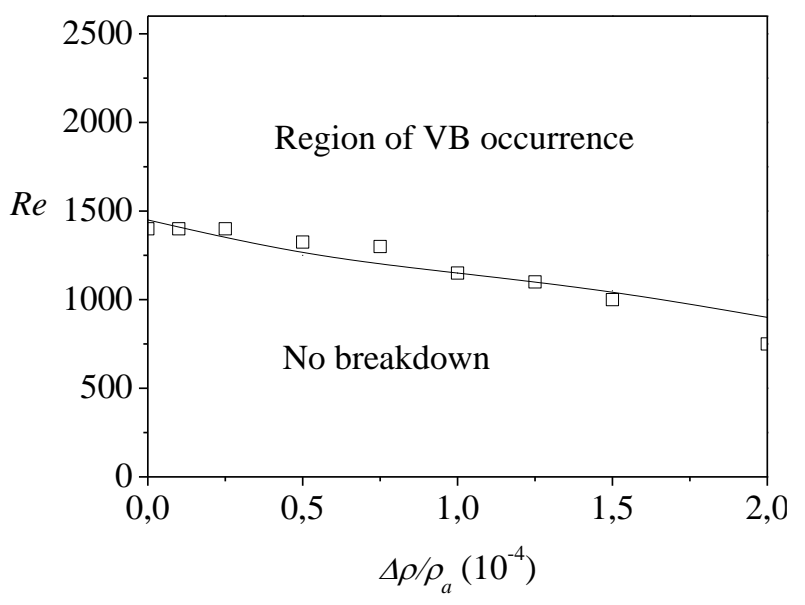

Fig. $2\left(R e, \Delta \rho / \rho_{a}>0\right)$ diagram, mapping regions of vortex breakdown (VB) occurrence under effect of heavy dye injection. Solid curve is the bounding curve predicted numerically and ( $\square$ ) Symbols from experiments [13]

\section{Results and discussion}

\subsection{Isothermal vortex flows under injection}

In this section is described briefly the incompressible swirling flow driven by the lid uniform rotation of a cylindrical enclosure with and without neutrally buoyant injection. As the study focused on axisymmetric structures which asymptote steady states, the main parameters were varied over the ranges $0.8 \leq \Lambda_{h} \leq 2.5,250 \leq R e \leq 2600$. We recall that outside the prescribed range of $R e$, the flow becomes time dependent (bubble oscillations evidenced by 3D simulations along the cavity axis [20]).

\subsubsection{Basic flow driven by the top disk rotation}

The model swirling flows under consideration, may sufficiently be described by two main parameters; namely, the rotational Reynolds number $R e$ and the cavity aspect ratio $\Lambda_{h}$. Qualitatively, for given low rates of top disk rotation, a clockwise large meridian circulation takes place which superimposes to the primary rotational motion. In particular, fluid spirals outward (inward) in the vicinity of the top rotating disk (still bottom disk) with the formation of a thin boundary layer on each disk. Along the cylindrical sidewall, flow is directed downward while the bulk flow is driven axially upward by the Ekman suction effect. Depending on the aspect ratio $\Lambda_{h}\left(\Lambda_{h}>1.2\right)$, as the disk rotation rate increases the flow stagnates at the cavity axis and an adverse pressure gradient takes place; giving rise to one, two or three reverse flows; commonly referred to as bubble type vortex breakdown. In the current work, the onset and location of a single bubble is evidenced numerically and viewed qualitatively (Fig.3) by the close up of selected meridian streamlines obtained for the couple of parameters $\left(\Lambda_{h}\right.$, $R e)=(1.98,1700)$, in good agreement with Escudier's experiments [1]. The figure also depicts the upstream and downstream stagnation points location bounding the bubble. Within this latter, meridian motion is anticlockwise and of weak intensity compared to the ambient clockwise primary 
circulation. The direction of the meridian motion within and outside the bubble is independent on that of the top disk rotation.

\subsubsection{Effect of neutrally buoyant injection}

Prior to investigating thermal effects, it appeared necessary to first consider the case under isothermal injection (neutrally buoyant injection $R i=0$ ) in order to assess the sensitivity of the vortex pattern to the inlet geometry and associated injection conditions.

Fig. 3 shows a close up of the meridian vortex pattern, before and after neutral injection applied to the above configuration $\left(\Lambda_{h}, R e\right)=(1.98,1700)$; assuming experimental conditions $[13,15]$ : uniform injection velocity $U_{j}=1.7 \times 10^{-3}$ $\mathrm{m} / \mathrm{s}$ at the orifice (radius ratio $\delta_{o}=R_{o} / R=7.69 \times 10^{-3}$ ). The figure reveals mainly a slight divergence of the selected streamlines in the vicinity of the axis, indicating a very weak flow deceleration downstream the vortex; causing a minor upward shift of the downstream stagnation point $P_{r}(<1 \%)$. However, these changes did not impact significantly the vortex topology and maximum vortex intensity $\left(\left|\psi_{\text {min }}\right|\right)$.
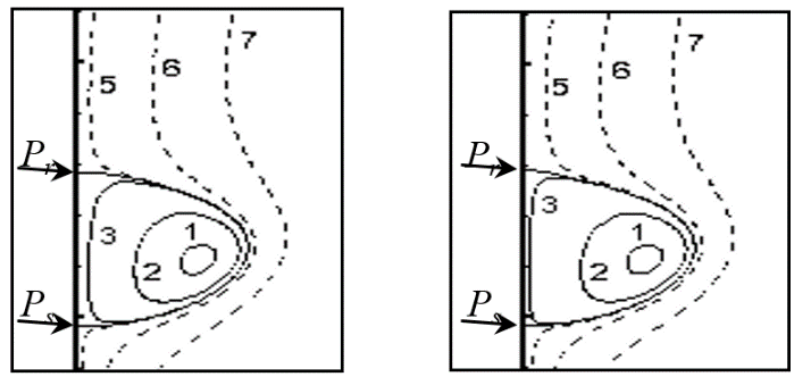

Fig. 3 A close up of an on-axis vortex (solid lines) in the meridian plane $[0, r[\times[0, z[$; (left) no injection and (right) under isothermal injection. $P_{s}$ and $P_{r}$ and are the upstream and downstream stagnation points respectively

\subsection{Effect of thermally buoyant injection}

In this section we focus on the case when the vortex flows are subjected to buoyancy, induced by uniform injection at temperature $T_{j}$ slightly different from the ambient fluid temperature $T_{a}$. The inlet conditions are the same as those implemented in the case of a neutrally buoyant injection $(R i=0)$ described above. Buoyancy strength is controlled by varying the Richardson number $R i$ over a range corresponding to small temperature gradients. Calculations were performed over the parameter ranges $250 \leq R e \leq 2600,0.8 \leq \Lambda_{h} \leq 2.5,-0.023 \leq R i \leq 0.092$.

\subsubsection{Warm injection}

When the jet temperature is slightly higher than that of the ambient fluid $(R i>0)$, calculations revealed significant changes of the flow pattern due to buoyancy. In particular, as depicted in Fig. $4 \mathrm{~b}$ for the selected parameters $(R e$, $\left.\Lambda_{h}, R i\right)=(1700,1.98,0.023)$ including the neutrally buoyant case $R i=0$ (Fig.4a), the on-axis stagnation points are suppressed while the bubble evolved into a ring type vortex, which remained robust, characterized by a saddle point at its periphery alongside with a stagnation point located at its center. These findings are qualitatively very consistent with the experimental results performed under light dye injection [13]. However, by varying $R i$ over the range $0 \leq R i \leq 0.092$, for fixed aspect ratio and $R e$ detailed time wise evolution of the flow field revealed the existence of a limited sub-region, involving relatively lower $R e$ values (state diagrams in the next section) where the ring type vortex itself continued to evolve by shrinking until it utterly disappeared; probably as a result of viscous damping. This trend is readily apparent in Fig. $4 \mathrm{c}$ for $\left(R e, \Lambda_{h}, R i\right)=(1700$, $1.98,0.046)$, showing a bulged region (swelling streamlines) which lacks breakdown. This numerically predicted flow evolution was found to occur for various couples of parameters $\left(R e, \Lambda_{h}, R i\right)$ as discussed in the next section. However, the experimental counterpart [13], performed for a single cavity aspect ratio, reported the bubble transition toward the ring type vortex but did not invoke the particular cases of its suppression; although one would expect, approximately, very similar trends as regards to the very small density variations involved in both studies. Analogous discrepancies between dye experiments on confined vortex breakdown and numerical predictions were reported by Herrada et al. $[10,11]$. We conjecture that such a discrepancy may mainly be attributed to the inevitable difference between the welldefined boundary conditions implemented in the frame work of axisymmetric simulations and the effective set up and operating conditions in experiments $[14,15]$. In addition, as a result of local dye diffusion, experimental images $[9,13,15,19]$ show frequently bulged regions of concentrated dye which do not accurately capture the required detailed vortex characteristics (saddle points and associated rings are hardly visible).

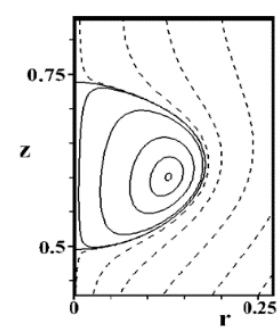

a) $R i=0$

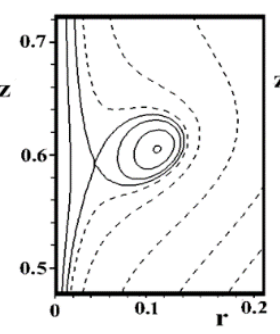

a) $R i=0.23$

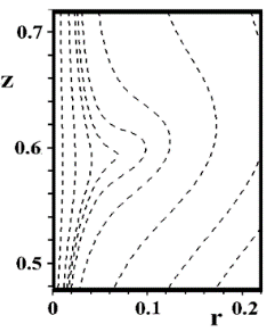

a) $R i=0.046$
Fig. 4 A close up of the vortex pattern (solid lines) under effect of warm injection (a), b), c)); $R e=1700$, $\Lambda_{h}=1.98$ and $R i$ as indicated

Combined effect of parameters $(R e, R i>0)$

The above findings are extended to explore the combined influence of the rotational Reynolds number and the buoyancy parameter $R i(R i>0)$. Results are summarized in the $R e(R i>0)$ state diagram (Fig. 5), established on the basis of detailed calculations carried out over the parameters ranges $1400 \leq R e \leq 2600$ and $0 \leq R i \leq 0.092$, for fixed cavity aspect ratio $\Lambda_{h}=1.98$ and two time stations: the higher time approaches steady state and the lower one was selected as a reference time. We recall that for $R e<1400$, the basic flow lacks breakdown.

The mapping identifies three distinct flow regimes with bounding curves, brought about by thermal effects. In particular, a region of bubble type (which shrinks with increasing time), another of robust toroidal eddy structure 
(which expands with time) and finally one which lacks breakdown, involving relatively lower $R e$ values and therefore more viscous damping. For fixed $R e$ over the range $1400<R e \leq 1750$, it is remarked that the bubble type vortex disappears under warm injection and the corresponding bounding curve is almost linear over the sub-range $0<R i<0.018$. Outside this latter interval, i.e. $0.018<R i<0.092$. variations are much less pronounced. We recall that the above numerical predictions showed that the bubble detached from the axis prior to its suppression. However, the remaining range $1700<R e<2600$ reveals that bubble bifurcation into a steady eddy structure, which remains observable, with increasing $R i$.

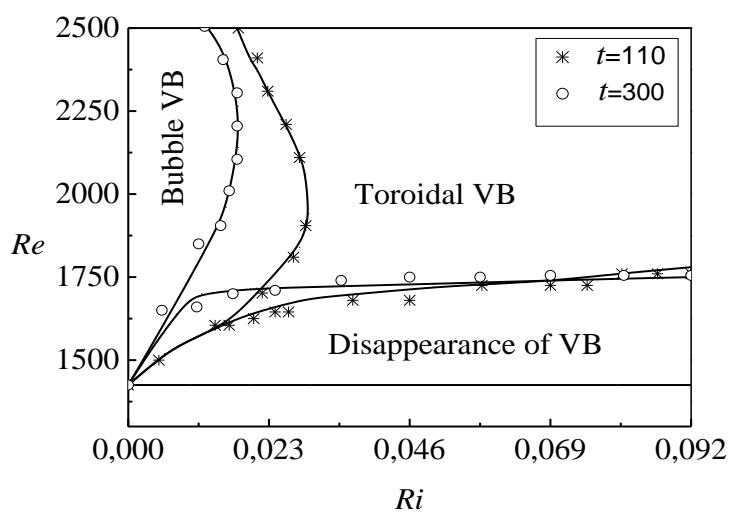

Fig. 5 ( $R e, R i>0)$ diagram for two selected times; as $R i$ increases vortex breakdown (VB) evolves into a toroidal pattern which may remain observable or disappear

The bounding curve, in this case, is almost linear over the approximate sub-range $1400<R e<2000$. Outside this latter range a decrease of $R i$ is remarked with increasing $R e$; indicating higher sensitivity of the bubble to the buoyant injection.

\subsubsection{Cool injection}

The model flow under cool injection $(R i<0)$, is described in this section. Unlike the previous case, buoyancy is shown here to promote flow stagnation conditions as well as vortex strength and size. This is evidenced in Fig. 6, for the selected couple of parameters $\left(R e, \Lambda_{h}, R i\right)=(1700$, $1.98, R i<0)$, which shows qualitatively, a more axially elongated and radially extended flow reversal than that resulting from the neutrally buoyant configuration. Besides, no ring type pattern was observed in this case.

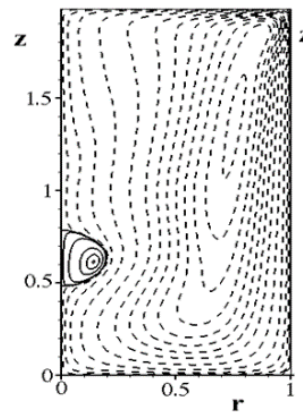

$R i=0$

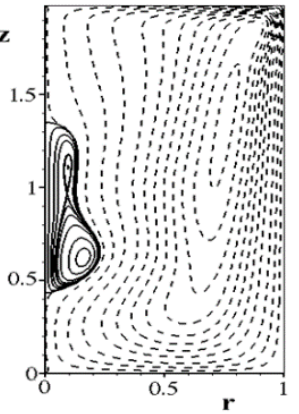

$R i=-0.023$
Fig. 6 Meridian streamlines illustrating effects of cool injection $(R i<0) ;\left(R e, \Lambda_{h}, R i\right)=(1700,1.98,-0.023)$

\subsection{3. $\left(R e, \Lambda_{h}\right)$ and $(R e, R i)$ state diagrams}

To further highlight the distinct and combined roles of the geometric and dynamic parameters involved, it appeared instructive and convenient to discuss the new $(R e$, $\left.\Lambda_{h}\right)$ and $(R e, R i)$ diagrams.

The effect of the cavity aspect ratio is illustrated in (Fig. 7) for $0.8 \leq \Lambda_{h} \leq 2.5,250 \leq R e \leq 2600$ and two selected $R i$ values; including the neutrally buoyant case $R i=0$ for comparison. For fixed $\Lambda_{h}$, the lower (upper) curve defines the critical $R e$ for breakdown onset (suppression). The case $R i=0$ (under injection) is approximately similar to the well documented configuration without injection [2]. Under a weak cool injection, the breakdown zone is shown to expand and cover wider ranges of $\left(R e, \Lambda_{h}\right)$. In particular, the lower bounding curves indicate a significant reduction of the threshold $\operatorname{Re}$ (up to 50\% reduction) for breakdown onset: much lower top disk rotation rates are sufficient to trigger flow stagnation. Besides, it is well established that configurations without injection display breakdown only if $\Lambda_{h}>1.3[1,2,19]$. In contrast, this limit is shown to reduce considerably when the flow is subjected to cool injection, as depicted in Fig. 8 over the range $0.8 \leq \Lambda_{h} \leq 1.3$.

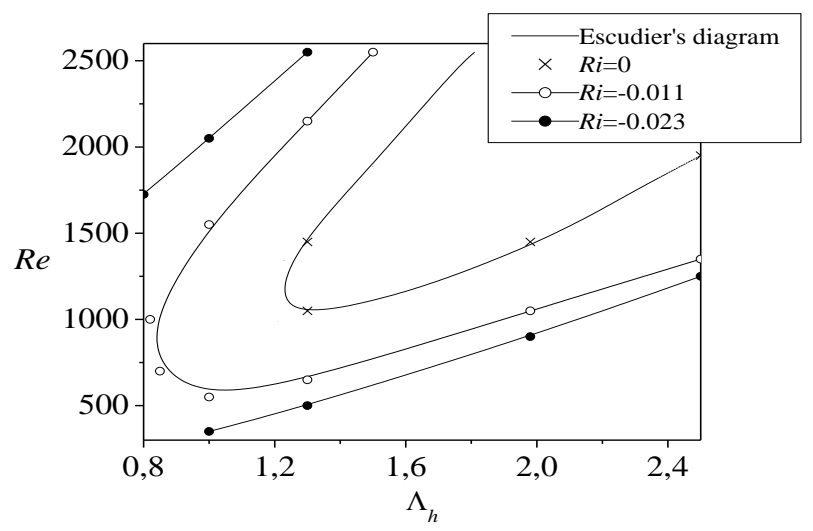

Fig. $7\left(R e, \Lambda_{h}\right)$ diagram mapping the breakdown region (bounded by an upper and lower curve) for three selected $R i<0$ values; cool injection enhances breakdown

Next, is discussed the sensitivity of breakdown to the combined variation of $R e$ and $R i$, for different aspect ratios, focusing on the impact of weak thermal buoyancy. Fig. 8 illustrates a $(R e, R i)$ diagram covering the ranges $250 \leq R e \leq 2600,-0.023 \leq R i \leq 0.023$ and for five distinct aspect ratios $\Lambda_{h}=1,1.3,1.64,1.98$ and 2.5 including the neutrally buoyant injection case $(R i=0)$ for the sake of comparison. It is clearly observed that $R i>0$ delays the flow stagnation as illustrated by the slight increase of the threshold $R e_{c}$ (by approximately $10 \%$ ) for $\Lambda_{h}>1.3$. However, weak buoyant cool injection $(R i<0)$, for all aspect ratios considered, is sufficient to promote breakdown occurrence as indicated by the steep decrease of the corresponding $R e_{c}$ bounding curves. The threshold $R e$ may drop by approximately $70 \%$ as depicted by comparing the trend of the lower curve corresponding to $\Lambda_{h}=1$ between the points $(R e \approx 1000, R i \approx-0.04)$ and $(R e \approx 300, R i \approx-0.023)$. Besides, it is remarked that under cool injection and over the range of $R e$ considered, cavities with $\Lambda_{h}=1$ and $\Lambda_{h}=1.3$ display breakdown in the inner region bounded by two 
curves while only a single threshold curve (lower bound) is illustrated for the three remaining relatively higher aspect ratios, the corresponding upper limit, if it exists, would probably occur over the range of oscillatory regime.

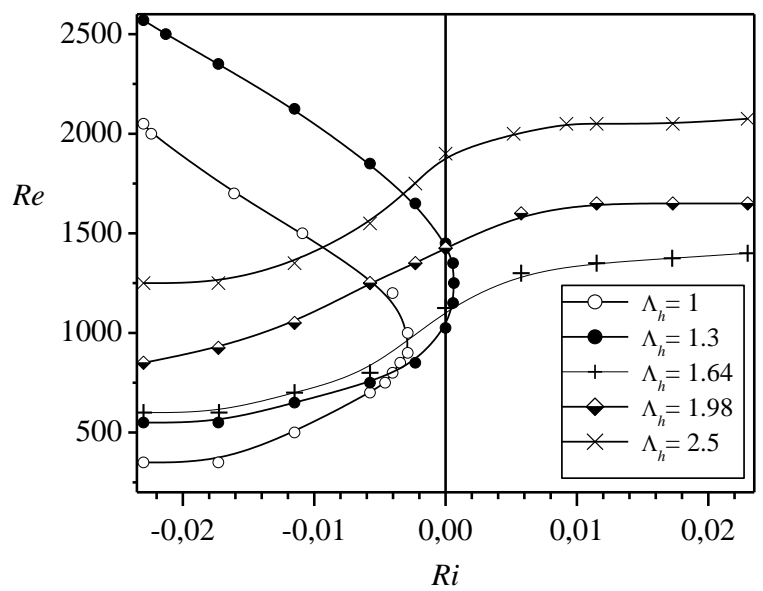

Fig. $8(R e, R i)$ diagram for five aspect ratios, indicating breakdown occurrence under warm $(R i>0)$ and cool ( $R i<0)$ injection: for $\Lambda_{h}=1$ and $\Lambda_{h}=1.3$ and breakdown bounded by an upper and lower curve while for higher aspect ratios it occurs above a single curve (breakdown threshold)

\subsubsection{The role of the local swirl number}

Breakdown in the disk-cylinder with spinning lid may be associated to the competition between two main local characteristics of the helical jet flow which develops within the viscous core region along the cavity axis $[6,21]$ namely the swirl strength and the meridian flow intensity characterized respectively by the local rotational and axial Reynolds numbers: $\quad R e_{r}=v_{m}^{*} r_{c}^{*} / v\left(R e_{r}=v_{m} r_{c} R e\right)$, $R e_{a}=w_{m}^{*} r_{c}^{*} / v\left(R e_{a}=w_{m} r_{c} R e\right)$, where variables with a star denote dimensional quantities. Here, $w_{m}=w_{\max }\left(0, z_{m}\right)$ is the maximum axial velocity reached at $z=z_{m}$ along the cavity axis and $v_{m}=v_{\max }\left(r_{c}, z_{m}\right)$ the maximum swirl velocity attained at the axial level $z=z_{m}$ and radial extent $r=r_{c}$ which defines the viscous core edge.

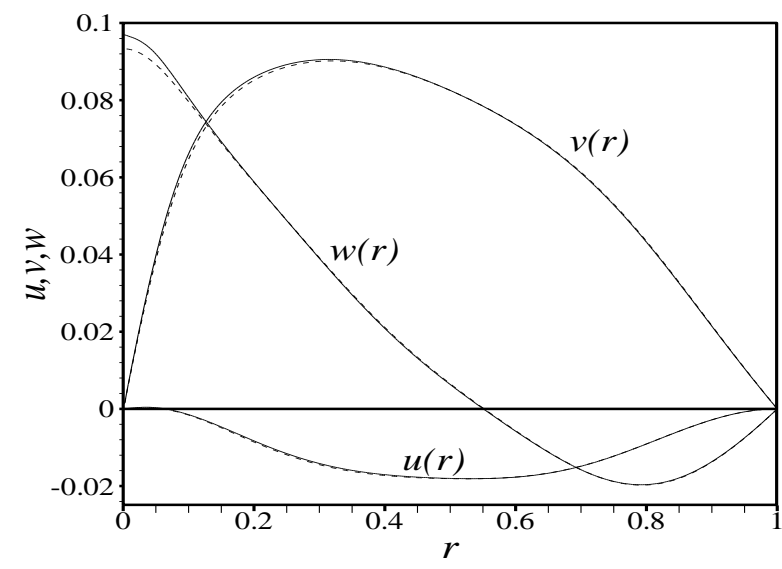

Fig. 9 Radial distribution of the velocity field at $z_{m}=0.26$ corresponding to the configuration $R e=2060, \Lambda_{h}=$ $=2.5, R i=0.023$ (solid curves) and $R i=0$ (broken curves)
Accordingly, a consistent local parameter to analyze breakdown development may therefore be constructed as the ratio $S_{N}=R e_{r} / R e_{a}$, which defines the local swirl number [6]. As a test case, the variation of $S_{N}$ is evaluated approximately in order to predict the sensitivity of the flow to warm injection for the selected configuration $R e=2060$, $H / R=2.5$ which displays two on-axis breakdown when $R i=$ 0 [1, 17, 19]. Calculations for this case indicated $z_{m}=0.26$, and the corresponding radial distribution of the velocity field (Fig. 9) provided $S_{N}=v_{m} / w_{m} \approx 0.97$ when $R i=0$ in agreement with recent numerical predictions [6]. Now, under warm injection $(R i=0.023)$ a small increase of $w_{m}$ is remarked due to buoyancy (the two remaining velocities are almost unaltered), which subsequently causes flow acceleration of weak intensity but sufficient to suppress breakdown. This effect corresponds to a swirl number reduction $\left(S_{N} \approx 0.93\right)$ just below the swirl strength required for breakdown onset. Besides, it can be remarked that the radial velocity magnitude is relatively small and its contribution to the local swirl number can be neglected.

\subsection{Differentially heated /cooled small rod}

In an attempt to confirm buoyancy influence on the stagnation flow conditions, the study was extended to consider a different set up which generates buoyancy by means of a differentially heated /cooled tiny rod sealed to the bottom disc center of the cylinder. Such a configuration and associated conditions are more amenable to numerical modeling and relatively easier to implement in practice.

This configuration introduces a modified Richardson number based on the rod temperature $\left(T_{\text {rod }}\right)$ which replace $\left(T_{j}\right)$. Besides, the rod radius $R_{r}$ is chosen to fit the orifice radius $R_{0}$ used in the injection case above $\left(R_{r}=R_{0}\right)$ which yields equal ratio $\delta_{r}=R_{r} / R=\delta_{0}\left(\delta_{o}=7.69 \times 10^{-3}\right.$ [13]). In order to obviate the occurrence of undesired additional eddies occurring at the rod tip which may alter breakdown onset conditions, we set the rod aspect ratio to $\delta_{h}=R_{r} / h=0.25$. Under such geometrical characteristics the presence of the rod did not alter significantly the vortex flows characteristics prior to differential heating and so may be regarded as a non-intrusive means of flow control. Qualitatively, the numerical predictions revealed that small temperature gradients corresponding to the range $-0.116 \leq R i \leq 0.116$, applied between the rod and the ambient fluid, were sufficient to alter significantly the vortex patterns. In particular, when the rod temperature is set slightly higher than that of the ambient fluid $(R i>0)$ a buoyancy force is generated, inducing an upward convection of swirling co-flow, of week intensity but sufficient to enhance the axial flux and angular momentum transfer, which act to prevent flow stagnation and associated vortex formation. This is depicted qualitatively, for $\Lambda_{h}=1.98, R e=1700$ and for the selected value $R i=0.116$, by the non-uniformly spaced meridian streamlines (Fig. 10, b); the case $R i=0$ (Fig.10, a) corresponds to the isothermal configuration. In contrast, when the rod is differentially cooled, an enhancement of the bubble size and occurrence of a second reverse flow structure were obtained (Fig. 10, c). 
In addition, to allow comparison of the threshold buoyancy parameter $R i$ required for breakdown suppression, in both rod and injection models, the axial velocity distribution at the cavity axis is supplied (Fig. 11), before and after thermal control, when $R=1700, \Lambda_{h}=1.98$. The figure confirms that prior to differential heating both means induce local effects without altering significantly the flow stagnation location. However, for a fixed ambient fluid temperature, to suppress breakdown by means of the rod differential heating, a temperature gradient $\Delta T=T_{\text {rod }}-T_{a}=5 K(R i=0.116)$ was required and no transient ring type vortex was found to occur. In contrast, calculations in the case of the injection model revealed that $\Delta T=1 K(R i=0.023)$ caused the detachment of the axial bubble which evolved into a robust steady ring type vortex while $\Delta T=2 K$ led a state which lacks breakdown.

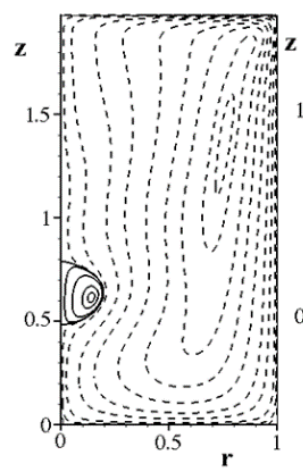

a) $R i=0$

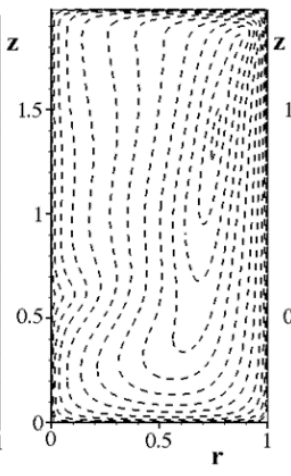

a) $R i=0.116$

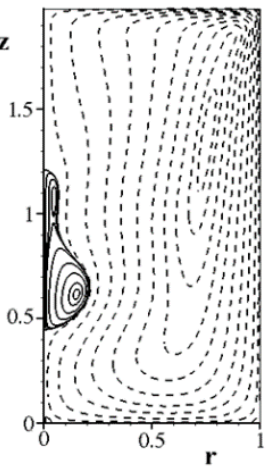

a) $R i=-0.116$
Fig. 10 Meridian flow pattern subjected to differentially heated/cooled rod; $R e=1700, \Lambda_{h}=1.98$

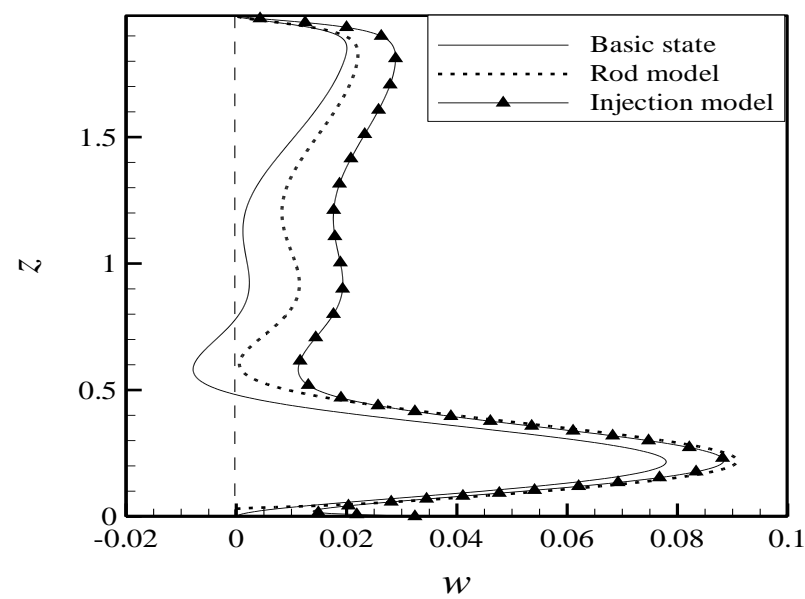

Fig. 11 Axial velocity distribution corresponding to the critical $R i$ required to suppress breakdown: $R i=0.116$ (rod case) and $R i=0.046$ (injection case); $R e=$ $=1700, \Lambda_{h}=1.98$

\section{Conclusion}

Thermal buoyancy, implemented by means of warm/cool uniform injection or by a differentially heated tiny rod, was shown numerically to constitute an effective means of controlling axial flow stagnation and associated breakdown in a disk-cylinder system with a rotating lid. In particular, warm (cool) injection or differentially heated (cooled) rod, applied upstream the vortex, were found to both prevent (favor) breakdown. In addition, under warm injection, buoyancy was found to suppress on-axis stagnation points while the associated bubble bifurcates into an off-axis vortex ring which may remain robust and observable or may continue to evolve until it disappears; depending on buoyancy strength and viscous damping effects. These findings were established for various $\left(\Lambda_{h}, R e, R i>0\right)$ parameters. Besides, the differentially heated/cooled tiny rod configuration, considered as a non-intrusive means based on thermal stratification was found to alter significantly the conditions of breakdown onset but, unlike the injection model, did not exhibit any ring type vortex. The current findings, which may be useful for bioreactors, constitute a platform for further investigations which, in perspective, explore oscillatory regimes.

\section{References}

1. Escudier, M. P. 1984. Observations of the flow produced in a cylindrical container by a rotating end wall, Exp. Fluids 2: 189-196.

http://dx.doi.org/10.1007/bf00571864.

2. Lopez, J. M. 1990. Axisymmetric vortex breakdown, Part 1, confined swirling flow, Journal of Fluid Mechanics 221: 533-552.

http://dx.doi.org/10.1017/s0022112090003664.

3. Mullin, T.; Kobine, J. J.; Tavener, S. J.; Cliffe, K. A. 2000. On the creation of stagnation points near straight and sloped walls, Physics of Fluids 12: 425-431.

http://dx.doi.org/10.1063/1.870320.

4. Mununga, L.; Lo Jacono, D.; Sørensen, J. N.; Leweke, T.; Thompson, M. C.; Hourigan, K. 2013. Control of confined vortex breakdown with partial rotating lids, Journal of Fluid Mechanics 738: 5-33. http://dx.doi.org/10.1017/jfm.2013.596.

5. Naumov, I. V.; Podolskaya, I. Y. 2017. Topology of vortex breakdown in closed polygonal containers, Journal of Fluid Mechanics 820: 263-283. http://dx.doi.org/10.1017/jfm.2017.211.

6. Jones, M. C.; Hourigan, K.; Thompson, M. C. 2015. Study of the geometry and parameter dependence of vortex breakdown, Physics of Fluids 27: 044102. http://dx.doi.org/10.1063/1.4916352.

7. Shtern, V. N. 2019. Mechanism of counterflows and circulation cells in swirling flows, Journal of Physics, conference series 1382012041 . http://dx.doi.org/10.1088/1742-6596/1382/1/012041.

8. Lugt, H. J.; Abboud, M. 1987. Axisymmetric vortex breakdown with and without temperature effects in a container with a rotating lid, Journal of Fluid Mechanics 179: 179-200. http://dx.doi.org/10.1017/s0022112087001484.

9. Omi, Y.; Iwatsu, R. 2005. Numerical study of swirling flows in a cylindrical container with co-/counter-rotating end disks under stable temperature difference, International Journal of Heat and Mass Transfer 48: 4854-4866. http://dx.doi.org/10.1016/j.ijheatmasstransfer.2005.05.038.

10. Herrada, M. A.; Shtern, V. 2003a. Control of vortex breakdown by temperature gradients, Physics of Fluids 15: 3468-3477. http://dx.doi.org/10.1063/1.1613646.

11. Herrada, M. A.; Shtern, V. 2003b. Vortex breakdown 
control by adding near-axis swirl and temperature gradients, Physical Review E 68: 041202.

http://dx.doi.org/10.1103/physreve.68.041202.

12. Dina, M.; Jacob, C. 2007. The effect of buoyancy on vortex breakdown in a swirling jet, J. Fluid Mech., 571: 177-189. http://dx.doi.org/10.1017/s0022112006002862.

13. Ismadi, M. Z.; Meunier, P.; Fouras, A.; Hourigan, K. 2011. Experimental control of vortex breakdown by density effects, Physics of Fluids 23: 034104. http://dx.doi.org/10.1063/1.3560386.

14. Brons, M.; Shen, W. Z.; Sorensen, J. N.; Zhu, W. J. 2007. The influence of imperfections on the flow structure of steady vortex breakdown bubbles, Journal of Fluid Mechanics 578: 453-466. http://dx.doi.org/10.1017/s0022112007005101.

15. Meunier, P.; Hourigan, K. 2013. Mixing in a vortex breakdown flow, Journal of Fluid Mechanics 731: 195222.

http://dx.doi.org/10.1017/jfm.2013.226.

16. Marugán-Cruz, C.; Rodríguez-Rodríguez, J.; Martínez-Bazán, C. 2013. Formation regimes of vortex rings in negatively buoyant starting jets, Journal of Fluid Mechanics 716: 470-486. http://dx.doi.org/10.1017/jfm.2012.554.

17. Dennis, D. J. C.; Seraudie, C.; Poole, R. J. 2014. Controlling vortex breakdown in swirling pipe flows: Experiments and simulations, Physics of Fluids 26 (5): 053602. http://dx.doi.org/10.1063/1.4875486.

18. Escudier, M. P.; O'Leary, J.; Poole, R. J. 2007. Flow produced in a conical container by a rotating endwall, Journal of Heat and Fluid Flow 28: 1418-142. http://dx.doi.org/10.1016/j.ijheatfluidflow.2007.04.018.

19. Iwatsu, R.; Koyama, S. A. 2007. A resolution independent solution for confined axisymmetric vortex breakdown flow, Journal of fluid science and technology 2: 215-225. http://dx.doi.org/10.1299/jfst.2.215.

20. Gelfgat, A. Y.; Bar-Yoseph, P. Z.; Solan, A. 2001. Three-dimensional instability of axisymmetric flow in a rotating lid-cylinder enclosure, Journal of Fluid Mechanics 438: 363-377.

http://dx.doi.org/10.1017/s0022112001004566.

21. Moise, P.; Mathew, J. 2019.Bubble and conical forms of vortex breakdown in swirling jets, Journal of Fluid Mechanics 853: 322-357.

http://dx.doi.org/10.1017/jfm.2019.401.

\author{
M. Fekhar, R. Saci, R. Gatignol
}

\section{SWIRLING FLOWS CHARACTERISTICS IN A CYLINDER UNDER EFFECT OF BUOYANCY}

S u m m a r y

Thermal buoyancy, induced by injection or by differential heating of a tiny rod is explored to control breakdown in the core of a helical flow driven by the lid rotation of a cylinder. Three main parameters are required to characterize numerically the flow behavior; namely, the rotational Reynolds number $R e$, the cavity aspect ratio $\Lambda_{h}$ and the Richardson number $R i$. Warm injection/rod, $R i>0$, is shown to prevent on-axis flow stagnation while breakdown enhancement is evidenced when $R i<0$. Results revealed that a bubble vortex evolves into a ring type structure which may remain robust, as observed in prior related experiments or, in contrast, disappear over a given range of parameters $\left(\Lambda_{h}\right.$, $R e, R i>0)$. Besides, the emergence of such a toroidal mode was not found to occur under thermal stratification induced by a differentially heated rod. Moreover, three state diagrams were established which provide detailed flow characteristics under the distinct and combined effects of buoyancy strength, viscous effects and cavity aspect ratio.

Keywords: breakdown, buoyancy, cylinder, injection, rotating lid, differential heating.

Received November 06, 2020 Accepted June 02, 2021

This article is an Open Access article distributed under the terms and conditions of the Creative Commons Attribution 4.0 (CC BY 4.0) License (http://creativecommons.org/licenses/by/4.0/). 